\title{
Genome-wide Analysis of Circular RNAs and Validation of hsa_circ_0086354 As a Promising Biomarker for Early Diagnosis of Cerebral Palsy
}

\section{Yuanyuan $\mathrm{Hu}$}

Xijing University

Xuzhao Bian

Xi'an Jiaotong University

Chao Wu

People's Hospital of Shapingba District

\section{Yan Wang}

Xi'an International Medical Center Hospital

\section{Yang Wu}

Xi'an International Medical Center Hospital

Shiquan Sun ( $\nabla$ sunsqxajt@163.com )

Xi'an Jiaotong University

\section{Research Article}

Keywords: cerebral palsy diagnosis, biomarker, hsa_circ_0086354

Posted Date: June 28th, 2021

DOI: https://doi.org/10.21203/rs.3.rs-598443/v1

License: (c) (i) This work is licensed under a Creative Commons Attribution 4.0 International License. Read Full License 


\section{Abstract}

Background: Cerebral palsy (CP) is a spectrum of non-progressive motor disorders caused by brain injury during fetal or postnatal periods. Current diagnosis of CP mainly relies on neuroimaging and motor assessment. Here, we aimed to explore novel biomarkers for early diagnosis of $\mathrm{CP}$.

Methods: Blood plasma from five CP children and their healthy twin brothers/sisters was analyzed by gene microarray to screen out differentially expressed RNAs. Selected differentially expressed circular RNAs (circRNAs) were further validated using quantitative real-time PCR. Receiver operating characteristic (ROC) curve analysis was used to evaluate the value of using hsa_circ_0086354 as a biomarker of CP.

Results: 43 up-regulated circRNAs and 2 down-regulated circRNAs were obtained by difference analysis (fold change $>2, p<0.05$ ), among which five circRNAs related to neuron differentiation and neurogenesis were chosen for further validation. Additional 30 pairs of CP children and healthy controls were recruited and five selected circRNAs were further detected, showing that hsa_circ_0086354 was significantly downregulated in CP plasma compared with control, which was highly in accord with microarray analysis. ROC curve analysis showed that the area under curve (AUC) to discriminate CP children and healthy controls using hsa_circ_0086354 was 0.967 , the sensitivity was 0.833 and the specificity was 0.966 . Moreover, hsa_circ_0086354 was predicted as a competitive endogenous RNA for miR-181a, miR-4741 and miR4656, and much literature evidence suggested that miR-181a may be a key target of hsa_circ_0086354 to regulate neuronal survival and neuronal differentiation.

Conclusion: Hsa_circ_0086354 was significantly down-regulated in blood plasma of CP children, which may be a novel competent biomarker for early diagnosis of CP.

\section{Background}

Since W.J. Little first described in the 1840s, the concept of cerebral palsy (CP) has been revised for several times and is now defined as a non-progressive motor disorder induced by brain injury during prenatal $(80 \%)$, perinatal $(10 \%)$ or postnatal $(10 \%)[1,2]$. The incidence of CP is 1.25 per 1000 neonates in China and 2-3 per 1000 neonates worldwide, and with a slight increase in the last two decades owing to the improvement of premature infant survival[3-5]. The brain injury in CP children results in activity limitation in most cases, accompanying with impaired communication and cognition.[6, 7]. To date, CP has no cure and would cost millions of healthcare expenditure, making $\mathrm{CP}$ as a severe public health problem that brings enormous burden for patient families $[2,8]$. Preterm birth and asphyxia result from dystocia are the most common risk factors for $\mathrm{CP}[9,10]$. Administration of magnesium sulfate for women at risks of premature delivery and cooling therapy for infants at high risks of $\mathrm{CP}$ are considered to be effective preventive methods [11-13]. Unfortunately, existing diagnosis by comprehensive analysis of neonatal encephalopathy history, neuroimaging and neurodevelopmental assessment is limited and needs further researches $[4,14]$. Therefore, a better understanding of CP aetiology and pursuit of more accurate early diagnostic methods are of great importance. 
Noncoding RNAs represents more than $98 \%$ of all human transcripts, among which circular RNAs (circRNAs) are a special subtype without $5^{\prime}$ cap or $3^{\prime}$ poly-A tail $[15,16]$. CircRNAs become a new research hotspot in the past decade owing to their diverse physiological functions: circRNAs sponge microRNAs according to the "competing endogenous RNA" (ceRNA) [17]; they also act as protein scaffolds or templates for protein translation $[18,19]$. Besides, increasing evidence indicates that circRNAs are implicated in the regulation of various human diseases including cardiovascular diseases, cancers and neurological diseases [20-22]. CircRNAs may also serve as potent biomarkers for the early detection of specific diseases attributing to its stability and easy accessibility [23, 24].

With the rapid development of next-generation sequencing, over 1000 circRNAs in human serum exosomes were identified [24, 25]. In present study, we screened out differential expressed circRNAs between $\mathrm{CP}$ children and their normal controls using microarray technology, to select novel biomarkers for early diagnosis and intervention of $\mathrm{CP}$ as well as provide a better understanding of $\mathrm{CP}$ etiology.

\section{Methods}

\section{Sample preparation}

Five CP children and their healthy twins were selected in our study to exclude interfering factors (Average age: $3.3 \pm 1.5$, average birth weight: $2.9 \pm 0.4 \mathrm{~kg}$ ). Additional 30 pairs of $\mathrm{CP}$ children and healthy controls were recruited for subsequent validation of differential expressed circRNAs (Age: $4.2 \pm 1.6$; average birth weight: $3.1 \pm 0.6 \mathrm{~kg}$ ). Inclusive criteria: 『undergo no drug therapy; $\otimes$ with complete clinical information; Exclusive criteria: $₫$ acute/chronic infectious diseases, connective tissue diseases or malignant tumor; $\otimes$ recent use of immunosuppressant; $\otimes$ injury of liver and kidney function. Whole blood sample $(5 \mathrm{ml}$ per patient) was collected in $\mathrm{Na}_{2}$ EDTA tubes. Plasma was isolated by centrifugation, followed by total RNA extraction using TRIzol reagent (ThermoFisher Scientific, Waltham, MA, USA). All blood samples were collected with the consent of parents of CP children. And all experiments performed in this study were in accord with the ethical guidelines of the Declaration of Helsinki and approved by the Ethics Committee of Xi'an International Medical Center Hospital.

\section{Microarray analysis}

After RNA integrity assessment using Agilent Bioanalyzer 2100 (Agilent technologies, Santa Clara, CA, USA), total RNAs were used to generate biotinylated cRNAs. Then cRNAs were hybridized with Hybridization Slides (Agilent technologies, Santa Clara, CA, USA) in a Hybridization Oven at $65^{\circ} \mathrm{C}$ for 17 hours. Sides were scanned under a Microarray Scanner (Agilent technologies, Santa Clara, CA, USA) and raw data were obtained by the Feature Extraction software 10.7 (Agilent technologies, Santa Clara, CA, USA), followed by raw data normalization using Quantile algorithm. CirRNAs with a fold change $>2, p$ value $<0.05$ were selected for subsequent analysis. For CP etiology and biomarker investigations, five circRNAs regarding neuron differentiation and neurogenesis were chosen for further quantitative real-time PCR verification. 


\section{Quantitative real-time PCR}

Additional thirty pairs of $\mathrm{CP}$ children and their health controls were recruited to verify the differential expressed circRNAs screened by the microarray. In brief, total RNAs of plasma were extracted using UNIQ10 RNA extraction kit (Sangon Biotech, Shanghai, China) and reversely transcribed into cDNA using Maxima Reverse Transcriptase (ThermoFisher Scientific, Waltham, MA, USA). Then cDNAs were quantified using Fast qPCR Master Mix (High Rox) (Sangon Biotech, Shanghai, China) in an ABI Stepone plus PCR instrument. 18S ribosomal RNA was used as internal control and all data were analyzed using the $2^{-\Delta \Delta}$ method. Specific primers used for circRNAs detection were listed in Table.1.

\section{Statistical analysis}

Raw data acquired from Microarray Scanner was normalized using Quantile algorithm. Selected differential expressed circRNAs were presented as heatmap plots using R package "pheatmap". Data from quantitative real-time PCR was analyzed using the $2^{-} \Delta \Delta$ method and mean values were compared using unpaired t-test (Graphpad Prism 8.0, USA). All experiments were repeated for at least three times and $p$ values less than 0.05 were regarded as statistically significant.

\section{Results}

\section{Differentially expressed circRNAs screened by microarray}

In order to exclude additional interfering factors, the blood samples from five pairs of twin children (one $\mathrm{CP}$ and one health) were collected in our study. Sino human ceRNA array V3.0 which includes 53,625 human circRNAs was used to screen out differentially expressed circRNAs between the twins. Volcano plot showed that 134 circRNAs were differentially expressed in CP children compared to their healthy controls, among which 77 circRNAs were up-regulated and 57 were down-regulated (fold change $>2$, $\mathrm{p}<0.05$ ) (Figure 1A). After further flag-signal screening, 45 differentially expressed human circRNAs were obtained (fold change>2, $\mathrm{p}<0.05$ ). As listed in Table.2, 43 circRNAs were up-regulated and 2 circRNAs were down-regulated. Among them, we selected five differentially expressed circRNAs that were mainly involved in neuron differentiation and neurogenesis for further quantitative real-time PCR verification. As clustering analysis of heatmap shows, hsa_circ_0042123, hsa_circ_0083264, hsa_circ_0035127 and hsa_circ_0015069 were up-regulated in CP group while hsa_circ_0086354 was down-regulated versus the control (Figure 1B).

\section{Hsa_circ_0086354 is a potential biomarker for early diagnosis of CP}

Further quantitative real-time PCR validation showed that the fold changes of CP vs. Control were as follow: hsa_circ_0042123 was -2.067 (microarray: 2.003), hsa_circ_0083264 was -1.031 (microarray: 
2.039), hsa_circ_0035127 was -1.408 (microarray: 2.144), hsa_circ_0015069 was 1.76 (microarray: 2.113) and hsa_circ_0086354 was -6.15 (microarray: -3.676) (Figure 2A). The expression pattern of hsa_circ_0086354 validated by real-time PCR was highly in accord with that detected by microarray, showing that hsa_circ_0086354 was significantly down-regulated in CP group (Figure 2B). We further analyzed the potential value of hsa_circ_0086354 as a biomarker of CP early diagnosis using receiver operating characteristic (ROC) curve. ROC curve analysis showed that the area under the curve (AUC) to discriminate $\mathrm{CP}$ and healthy control using hsa_circ_0086354 level was 0.967 , the sensitivity was 0.833 and the specificity was 0.966 (Figure 2C), suggesting that hsa_circ_0086354 is a competent biomarker for CP diagnosis.

\section{Hsa_circ_0086354 is involved in neuronal survival and neuronal differentiation via targeting miR-181a}

Hsa_circ_0086354 associated ceRNA network was obtained using Cytoscape analysis. MiR-181a, miR4741 and miR-4656 were down-stream target microRNAs of hsa_circ_0086354 (Figure 3A), and further literature search indicated that miR-181a may be a key targeted regulator of hsa_circ_0086354 to be involved in the regulation of neuronal survival and neuronal differentiation (Figure 3B).

\section{Discussion}

Owing to its enigmatic etiology, the diagnosis of $\mathrm{CP}$ can barely rely on neuroimaging and assessment of motor dysfunction [26]. CirRNAs were first considered as byproducts of mis-splicing, yet increasing evidence indicated that circRNAs are implicated in various molecular processes as well as human diseases: circRNAs regulate gene expression via regulating gene transcription, gene splicing or sponging microRNAs; circRNAs are involved in the regulation of neuronal diseases, cardiovascular disease and cancer progression. Of note, ciRS-7 regulates a-synuclein expression through co-expressing and colocalizing with miR-7 to further regulate brain development [25]. Besides, majority of identified circRNAs are abundantly detected in brain tissues and neurons, which inspired us to explore specific biomarkers for CP diagnosis.

In the present study, blood samples from five CP children and their twin brothers/sisters were collected to screen out differentially expressed circRNAs using microarray. Twin participants at identical preterm conditions can exclude additional risk factors of $\mathrm{CP}$, which makes our results more reliable. Five circRNAs enriched in neuron differentiation and neurogenesis were selected from 45 differentially expressed circRNAs for further validation. Another 30 pairs of plasma samples from CP children and healthy controls were collected, and the expression levels of five selected circRNAs were quantified. It was remarkable that the expression pattern of hsa_circ_0086354 measured by quantitative real-time PCR was highly in consistent with that detected by microarray. Yet the expression differences between CP children and health controls of hsa_circ_0042123, hsa_circ_0083264, hsa_circ_0035127 and hsa_circ_0015069 were either not significant or contradictory with microarray analysis. Therefore, our findings suggest that hsa_circ_0086354 might serve as a promising biomarker for CP diagnosis. 
CircRNAs have been reported to serve as competent biomarkers for diagnosis of various diseases. For instance, plasma hsa_circRNA_002453 was a potential biomarker for severity of renal involvement and diagnosis of lupus nephritis with an AUC of 0.906 [27]. Hsa_circRNA_0000520 is remarkably downregulated in gastric cancer and may serve as a potential biomarker for early diagnosis [28]. Hsa_circRNA_0001649 is a novel specific biomarker for colorectal cancer assessment [29]. CircRNAs display high stability owing to their covalent loop structure, which helps them get rid of de-adenylation, de-capping and RNases degradation. The tissue-specific expression pattern of circRNAs enables them to serve as specific biomarkers for specific diseases [30,31]. The application of circRNAs as biomarkers has always been a controversial topic, and the abundance of circRNAs is the major concern. Indeed, generally, the abundance of circRNAs is relatively low compared to their linear RNA product in body fluids. However, others demonstrated that some circRNAs are detected at comparable, even higher expression to their linear RNA [32,33]. Besides, the rapid development of next-generation sequencing will provide substantial technical support for circRNA detection. Dong, R concluded that majority of annotated circRNAs are identified in brain tissues and neurons [34]. In the present study, hsa_circ_0086354 was significantly down-regulated in CP plasma with an AUC of 0.967, suggesting hsa_circ_0086354 may be a promising biomarker for the early diagnosis of CP. In addition, the host gene of hsa_circ_0086354 is protein tyrosine phosphatase receptor type $D$ (PTPRD), which is highly expressed in brain tissues and regulated neurite growth and neurons axon guidance, indicating that PTPRD and hsa_circ_0086354 might involve in CP etiology $[35,36]$.

We further discovered that hsa_circ_0086354 acts as a ceRNA of miR-181a. MiR-181a is up-regulated in patients with mild cognitive impairment which later progressed to Alzheimer's disease [37]. MiR-181a is also up-regulated in rats after ischemia/reperfusion induced cerebral injury [38]. On the contrary, miR181a silencing exerts neuroprotective effects through suppressing neuronal apoptosis and neuronal loss both in a rat model and in epilepsy children [39, 40]. MiR-181a silencing also promotes neuronal growth via regulating the Smad signaling in Parkinson's disease [41]. These previous findings imply that miR181a level is negatively correlated to neuronal survival, and down-regulation of hsa_circ_0086354 might inhibit neuronal growth through restoring miR-181a in CP children. Besides, miR-181a contributes to neural stem cell differentiation and promotes generation of neurons, indicating hsa_circ_0086354 may also be involved in neuronal differentiation through targeting miR-181a [42, 43].

\section{Conclusion}

Hsa_circ_0086354 is significantly down-regulated in CP children in contrast with their healthy control with an AUC of 0.967 , making it as a promising biomarker for the early diagnosis of CP. Hsa_circ_0086354 may also be involved in the etiology of $\mathrm{CP}$ through regulating neuronal survival and neuronal differentiation by targeting miR-181a.

\section{Abbreviations}

CP: cerebral palsy 
circRNAs: circular RNAs

ceRNA": competing endogenous RNA"

ROC: receiver operating characteristic

AUC: under the curve

PTPRD: protein tyrosine phosphatase receptor type D

\section{Declarations}

\section{Ethics approval and consent to participate}

All blood samples were collected with the consent of legal guardians of CP children. And all experiments performed in this study were in accord with the ethical guidelines of the Declaration of Helsinki approved by the Ethics Committee of Xijing university.

\section{Authors' contributions}

Conceptualization: Shiquan Sun and Xuzhao Bian; methodology: Yuanyuan Hu; formal analysis:

Yuanyuan Hu; Sample collection: Chao Wu and Yan Wang; writing-original draft preparation: Xuzhao Bian; writing-review and editing: Yang Wu; All authors have read and agreed to the published version of the manuscript.

\section{Consent for publication}

Written consent to publish this information was obtained from legal guardians of participants by the study coordinators. All legal guardians were informed about sample collection, data publication and personal information confidentiality.

\section{Availability of data and material}

The datasets generated and/or analysed during the current study are available in the Jianguoyun repository (https://www.jianguoyun.com/p/DeUBPbUQ4o7JCRjzzfkD).

\section{Competing interests}

The authors declare no conflict of interest.

\section{Funding}

Not applicable.

\section{Acknowledgements}


Not applicable.

\section{References}

1. Little, W. J. The classic: Hospital for the cure of deformities: course of lectures on the deformities of the human frame. 1843. Clin Orthop Relat Res 2012 470: 1252-6.

2. Sewell, M. D., Eastwood, D. M. \& Wimalasundera, N. Managing common symptoms of cerebral palsy in children. BMJ 2014 349: g5474.

3. He, P., Chen, G., Wang, Z. et al. Children with motor impairment related to cerebral palsy: Prevalence, severity and concurrent impairments in China. J Paediatr Child Health 2017 53: 480-484.

4. Korzeniewski, S. J., Slaughter, J., Lenski, M. et al. The complex aetiology of cerebral palsy. Nat Rev Neurol 2018 14: 528-543.

5. Bhushan, V., Paneth, N. \& Kiely, J. L. Impact of improved survival of very low birth weight infants on recent secular trends in the prevalence of cerebral palsy. Pediatrics 1993 91: 1094-100.

6. Sadowska, M., Sarecka-Hujar, B. \& Kopyta, I. Cerebral Palsy: Current Opinions on Definition, Epidemiology, Risk Factors, Classification and Treatment Options. Neuropsychiatr Dis Treat 2020 16: 1505-1518.

7. Pakula, A. T., Van Naarden Braun, K. \& Yeargin-Allsopp, M. Cerebral palsy: classification and epidemiology. Phys Med Rehabil Clin N Am 2009 20: 425-52.

8. Kancherla, V., Amendah, D. D., Grosse, S. D. et al. Medical expenditures attributable to cerebral palsy and intellectual disability among Medicaid-enrolled children. Res Dev Disabil 2012 33: 832-40.

9. Frank, R., Garfinkle, J., Oskoui, M. et al. Clinical profile of children with cerebral palsy born term compared with late- and post-term: a retrospective cohort study. BJOG 2017 124: 1738-1745.

10. Moster, D., Wilcox, A. J., Vollset, S. E. et al. Cerebral palsy among term and postterm births. JAMA 2010 304: 976-82.

11. Nelson, K. B. \& Grether, J. K. Can magnesium sulfate reduce the risk of cerebral palsy in very low birthweight infants? Pediatrics 1995 95: 263-9.

12. Schendel, D. E., Berg, C. J., Yeargin-Allsopp, M. et al. Prenatal magnesium sulfate exposure and the risk for cerebral palsy or mental retardation among very low-birth-weight children aged 3 to 5 years. JAMA 1996 276: 1805-10.

13. Rao, R., Trivedi, S., Vesoulis, Z. et al. Safety and Short-Term Outcomes of Therapeutic Hypothermia in Preterm Neonates 34-35 Weeks Gestational Age with Hypoxic-Ischemic Encephalopathy. J Pediatr 
14. Novak, I., Morgan, C., Adde, L. et al. Early, Accurate Diagnosis and Early Intervention in Cerebral Palsy: Advances in Diagnosis and Treatment. JAMA Pediatr 2017 171: 897-907.

15. Jeck, W. R. \& Sharpless, N. E. Detecting and characterizing circular RNAs. Nat Biotechnol 2014 32: 453-61.

16. Birney, E.Stamatoyannopoulos, J. A.Dutta, A. et al. Identification and analysis of functional elements in 1\% of the human genome by the ENCODE pilot project. Nature 2007 447: 799-816.

17. Salmena, L., Poliseno, L., Tay, Y. et al. A ceRNA hypothesis: the Rosetta Stone of a hidden RNA language? Cell 2011 146: 353-8.

18. Ashwal-Fluss, R., Meyer, M., Pamudurti, N. R. et al. circRNA biogenesis competes with pre-mRNA splicing. Mol Cell 2014 56: 55-66.

19. Pamudurti, N. R., Bartok, O., Jens, M. et al. Translation of CircRNAs. Mol Cell 2017 66: 9-21 e7.

20. Altesha, M. A., Ni, T., Khan, A. et al. Circular RNA in cardiovascular disease. J Cell Physiol 2019 234: 5588-5600.

21. Li, Y., Ge, Y. Z., Xu, L. et al. Circular RNA ITCH: A novel tumor suppressor in multiple cancers. Life Sci 2020 254: 117176.

22. Li, T. R., Jia, Y. J., Wang, Q. et al. Circular RNA: a new star in neurological diseases. Int J Neurosci 2017 127: 726-734.

23. Lei, B., Tian, Z., Fan, W. et al. Circular RNA: a novel biomarker and therapeutic target for human cancers. Int J Med Sci 2019 16: 292-301.

24. Li, Y., Zheng, Q., Bao, C. et al. Circular RNA is enriched and stable in exosomes: a promising biomarker for cancer diagnosis. Cell Res 2015 25: 981-4.

25. Hsiao, K. Y., Sun, H. S. \& Tsai, S. J. Circular RNA - New member of noncoding RNA with novel functions. Exp Biol Med (Maywood) 2017 242: 1136-1141.

26. Ashwal, S., Russman, B. S., Blasco, P. A. et al. Practice parameter: diagnostic assessment of the child with cerebral palsy: report of the Quality Standards Subcommittee of the American Academy of Neurology and the Practice Committee of the Child Neurology Society. Neurology 2004 62: 851-63.

27. Ouyang, Q., Huang, Q., Jiang, Z. et al. Using plasma circRNA_002453 as a novel biomarker in the diagnosis of lupus nephritis. Mol Immunol 2018 101: 531-538. 
28. Sun, H., Tang, W., Rong, D. et al. Hsa_circ_0000520, a potential new circular RNA biomarker, is involved in gastric carcinoma. Cancer Biomark 2018 21: 299-306.

29. Ji, W., Qiu, C., Wang, M. et al. Hsa_circ_0001649: A circular RNA and potential novel biomarker for colorectal cancer. Biochem Biophys Res Commun 2018 497: 122-126.

30. Salzman, J., Gawad, C., Wang, P. L. et al. Circular RNAs are the predominant transcript isoform from hundreds of human genes in diverse cell types. PLoS One 2012 7: e30733.

31. Salzman, J., Chen, R. E., Olsen, M. N. et al. Cell-type specific features of circular RNA expression. PLoS Genet 2013 9: e1003777.

32. Salzman, J. Circular RNA Expression: Its Potential Regulation and Function. Trends Genet 201632 : 309-316.

33. Jeck, W. R., Sorrentino, J. A., Wang, K. et al. Circular RNAs are abundant, conserved, and associated with ALU repeats. RNA 2013 19: 141-57.

34. Dong, R., Ma, X. K., Chen, L. L. et al. Increased complexity of circRNA expression during species evolution. RNA Biol 2017 14: 1064-1074.

35. Tomita, H., Cornejo, F., Aranda-Pino, B. et al. The Protein Tyrosine Phosphatase Receptor Delta Regulates Developmental Neurogenesis. Cell Rep 2020 30: 215-228 e5.

36. Uhl, G. R. \& Martinez, M. J. PTPRD: neurobiology, genetics, and initial pharmacology of a pleiotropic contributor to brain phenotypes. Ann N Y Acad Sci 2019 1451: 112-129.

37. Ansari, A., Maffioletti, E., Milanesi, E. et al. miR-146a and miR-181a are involved in the progression of mild cognitive impairment to Alzheimer's disease. Neurobiol Aging 2019 82: 102-109.

38. Zhang, Y., Shan, Z., Zhao, Y. et al. Sevoflurane prevents miR-181a-induced cerebral ischemia/reperfusion injury. Chem Biol Interact 2019 308: 332-338.

39. Ren, L., Zhu, R. \& Li, X. Silencing miR-181a produces neuroprotection against hippocampus neuron cell apoptosis post-status epilepticus in a rat model and in children with temporal lobe epilepsy. Genet Mol Res 2016 15:

40. Moon, J. M., Xu, L. \& Giffard, R. G. Inhibition of microRNA-181 reduces forebrain ischemia-induced neuronal loss. J Cereb Blood Flow Metab 2013 33: 1976-82.

41. Hegarty, S. V., Sullivan, A. M. \& O'Keeffe, G. W. Inhibition of miR-181a promotes midbrain neuronal growth through a Smad1/5-dependent mechanism: implications for Parkinson's disease. Neuronal Signal 2018 2: NS20170181. 
42. Stappert, L., Borghese, L., Roese-Koerner, B. et al. MicroRNA-based promotion of human neuronal differentiation and subtype specification. PLoS One 2013 8: e59011.

43. Hamada, N., Fujita, Y., Kojima, T. et al. MicroRNA expression profiling of NGF-treated PC12 cells revealed a critical role for miR-221 in neuronal differentiation. Neurochem Int 2012 60: 743-50.

\section{Tables}

Table 1 Primers used for quantitative real-time PCR in this study.

\begin{tabular}{|c|c|}
\hline Target & Primers \\
\hline \multirow[t]{2}{*}{ hsa_circ_0042123 } & Forward: 5'-TCAGCAACAGGAGGAGCATT-3' \\
\hline & Reverse: 5'-CCTCAGGAAATGTCCACCACT-3' \\
\hline \multirow[t]{2}{*}{ hsa_circ_0083264 } & Forward: 5'-AAGCCCATCCAGAGGTTCC-3' \\
\hline & Reverse: 5'-СTGTTCTСССТСТTССТСТTСАT-3' \\
\hline \multirow[t]{2}{*}{ hsa_circ_0035127 } & Forward: 5'-ТСТАТТСАТТССТССААААССТG-3' \\
\hline & Reverse: 5'-ATGGGAAGCGGAATGAGAG-3' \\
\hline \multirow[t]{2}{*}{ hsa_circ_0086354 } & Forward: 5'-ACTTGGGCTGGTGCAACTAA-3 \\
\hline & Reverse: 5'-GGCCCGGGCCATATAGT-3' \\
\hline \multirow[t]{2}{*}{ hsa_circ_0015069 } & Forward: 5'-ACTCGCAGCCAGTCAGATGTA-3' \\
\hline & Reverse: 5'-TGACTGCACGCTCATGAACA-3' \\
\hline \multirow[t]{2}{*}{ Hsa-18s rRNA } & Forward: 5'-GGACACGGACAGGATTGACA-3' \\
\hline & Reverse: 5'-CCAGAGTCTCGTTCGTTATCG-3' \\
\hline
\end{tabular}

Table 2. Microarray analysis of differential expressed circRNAs in $5 \mathrm{CP}$ children compared with their healthy twins. 


\begin{tabular}{|c|c|c|c|c|c|}
\hline CircRNA_ID & Regulation & Fold change & $P$ values & Circ_chromosome & Host gene \\
\hline hsa_circ_0062733 & up & 3.195 & 0.010 & chr22 & EMID1 \\
\hline hsa_circ_0066747 & up & 2.496 & 0.032 & chr3 & MYH15 \\
\hline hsa_circ_0030588 & up & 2.492 & 0.012 & chr13 & $\mathrm{ABCC} 4$ \\
\hline hsa_circ_0020792 & up & 2.488 & 0.048 & chr11 & INS-IGF2 \\
\hline hsa_circ_0007110 & up & 2.466 & 0.048 & chr9 & DENND4C \\
\hline hsa_circ_0049906 & up & 2.442 & 0.040 & $\operatorname{chr19}$ & HAUS8 \\
\hline hsa_circ_0031700 & up & 2.391 & 0.027 & chr14 & MIPOL1 \\
\hline hsa_circ_0036358 & up & 2.360 & 0.027 & chr15 & PTPN9 \\
\hline hsa_circ_0036730 & up & 2.350 & 0.021 & chr15 & C15orf42 \\
\hline hsa_circ_0066990 & up & 2.319 & 0.028 & chr3 & KPNA1 \\
\hline hsa_circ_0016754 & up & 2.267 & 0.032 & chr1 & CDC42BPA \\
\hline hsa_circ_0068412 & up & 2.257 & 0.025 & chr3 & IGF2BP2 \\
\hline hsa_circ_0087881 & up & 2.208 & 0.025 & chr9 & CTNNAL1 \\
\hline hsa_circ_0084683 & up & 2.186 & 0.039 & chr8 & CSPP1 \\
\hline hsa_circ_0042530 & up & 2.182 & 0.031 & chr17 & POLDIP2 \\
\hline hsa_circ_0035047 & up & 2.147 & 0.020 & chr15 & WDR76 \\
\hline hsa_circ_0035127 & up & 2.144 & 0.050 & chr9 & MYEF2 \\
\hline hsa_circ_0043970 & up & 2.136 & 0.044 & chr17 & NBR1 \\
\hline hsa_circ_0054449 & up & 2.133 & 0.020 & chr2 & EPAS1 \\
\hline hsa_circ_0068411 & up & 2.131 & 0.036 & chr3 & IGF2BP2 \\
\hline hsa_circ_0071500 & up & 2.128 & 0.039 & chr4 & WWC2 \\
\hline hsa_circ_0033776 & up & 2.126 & 0.038 & chr14 & None \\
\hline hsa_circ_0084682 & up & 2.123 & 0.036 & chr8 & CSPP1 \\
\hline hsa_circ_0071499 & up & 2.120 & 0.024 & chr4 & WWC2 \\
\hline hsa_circ_0087309 & up & 2.118 & 0.031 & chr9 & TLE1 \\
\hline hsa_circ_0090182 & up & 2.117 & 0.033 & $\operatorname{chr} X$ & PRRG1 \\
\hline hsa_circ_0036485 & up & 2.114 & 0.042 & chr15 & ADAMTS7 \\
\hline hsa_circ_0015069 & up & 2.113 & 0.004 & chr1 & PBX1 \\
\hline
\end{tabular}




\begin{tabular}{|llllll|} 
hsa_circ_0013249 & up & 2.087 & 0.030 & chr1 & TMEM56 \\
\hline hsa_circ_0087882 & up & 2.082 & 0.017 & chr9 & CTNNAL1 \\
hsa_circ_0071976 & up & 2.078 & 0.040 & chr5 & ANKH \\
hsa_circ_0087880 & up & 2.077 & 0.043 & chr9 & CTNNAL1 \\
hsa_circ_0009100 & up & 2.055 & 0.014 & chr17 & PRR11 \\
\hline hsa_circ_0030584 & up & 2.051 & 0.024 & chr13 & ABCC4 \\
\hline hsa_circ_0039989 & up & 2.046 & 0.012 & chr16 & CDH3 \\
\hline hsa_circ_0083264 & up & 2.039 & 0.006 & chr8 & ARHGEF10 \\
\hline hsa_circ_0056717 & up & 2.036 & 0.024 & chr2 & RIF1 \\
\hline hsa_circ_0045000 & up & 2.024 & 0.029 & chr17 & BCAS3 \\
\hline hsa_circ_0047155 & up & 2.015 & 0.028 & chr18 & RIOK3 \\
\hline hsa_circ_0087884 & up & 2.013 & 0.027 & chr9 & CTNNAL1 \\
\hline hsa_circ_0016274 & up & 2.007 & 0.022 & chr1 & YOD1 \\
\hline hsa_circ_0042123 & up & 2.003 & 0.002 & chr17 & PMP22 \\
\hline hsa_circ_0062335 & up & 2.002 & 0.029 & chr22 & PI4KA \\
\hline hsa_circ_0077792 & down & 0.492 & 0.027 & chr6 & TRMT11 \\
\hline hsa_circ_0086354 & down & 0.272 & 0.016 & chr15 & PTPRD \\
\hline
\end{tabular}

\section{Figures}


A

CP vs. Control

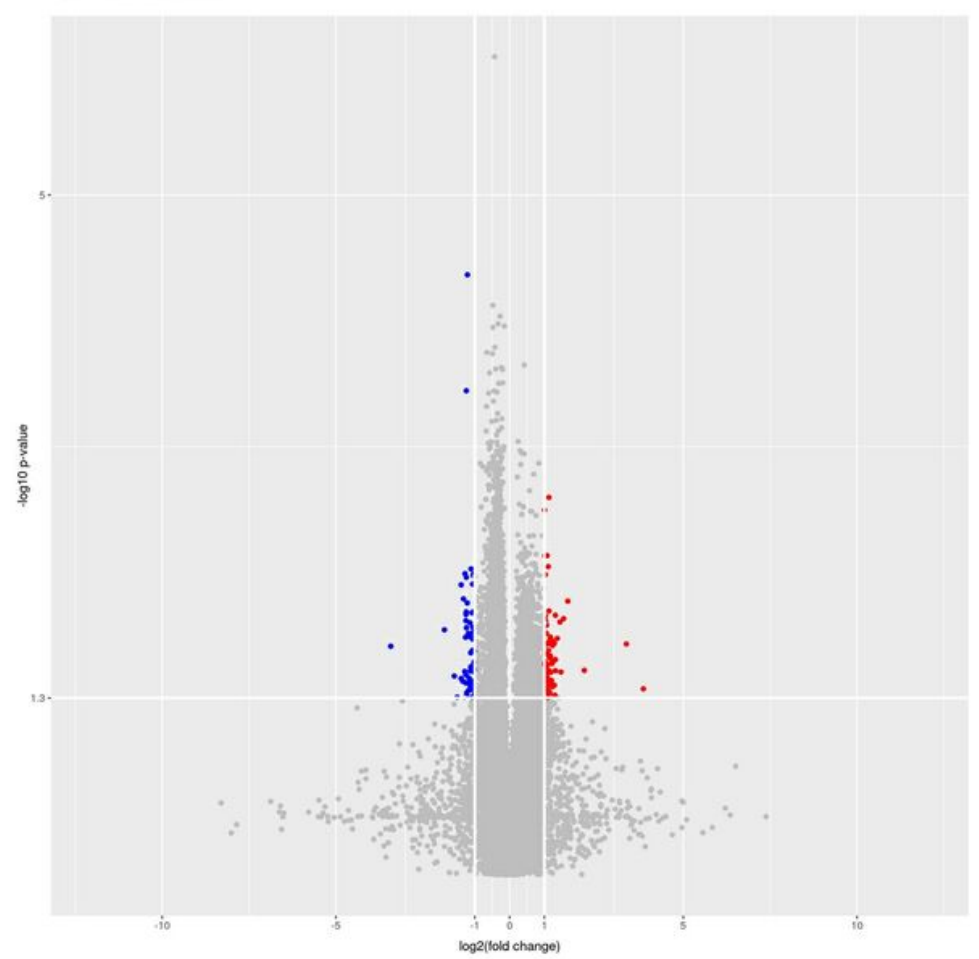

B

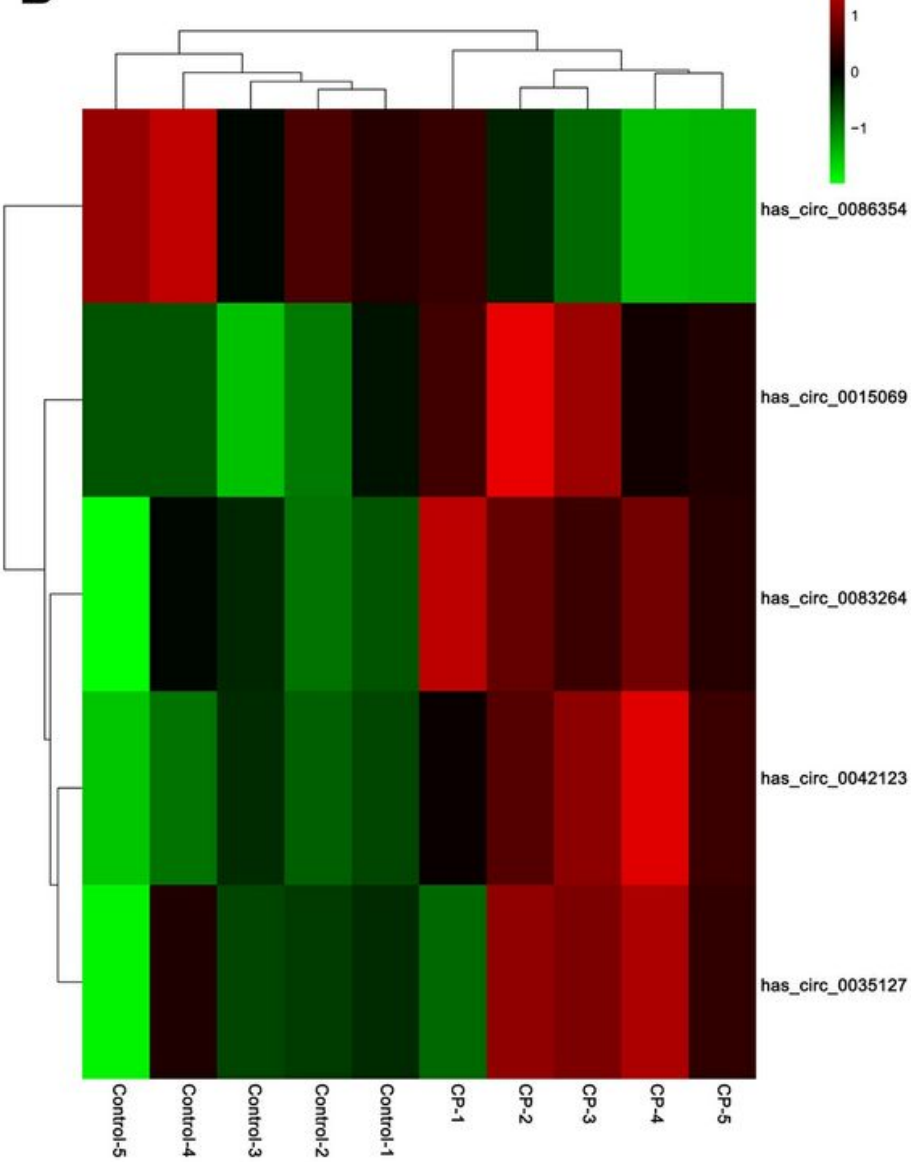

\section{Figure 1}

Differentially expressed circRNAs between CP children and their health twins. (A) Differentially expressed circRNAs between CP children and their health controls was shown in Volcano plot. "Red" represents upregulated circRNAs, "Blue" represents down-regulated circRNAs. (fold change $>2, p<0.05$ ) (B) Heatmap clustering analysis was performed to display 5 selected circRNAs. Rows represent differential circRNAs and columns represent five pairs of CP samples and healthy controls. "Green" represents down-regulation and "Red" represents up-regulation of circRNAs in each sample. CP: cerebral palsy. 


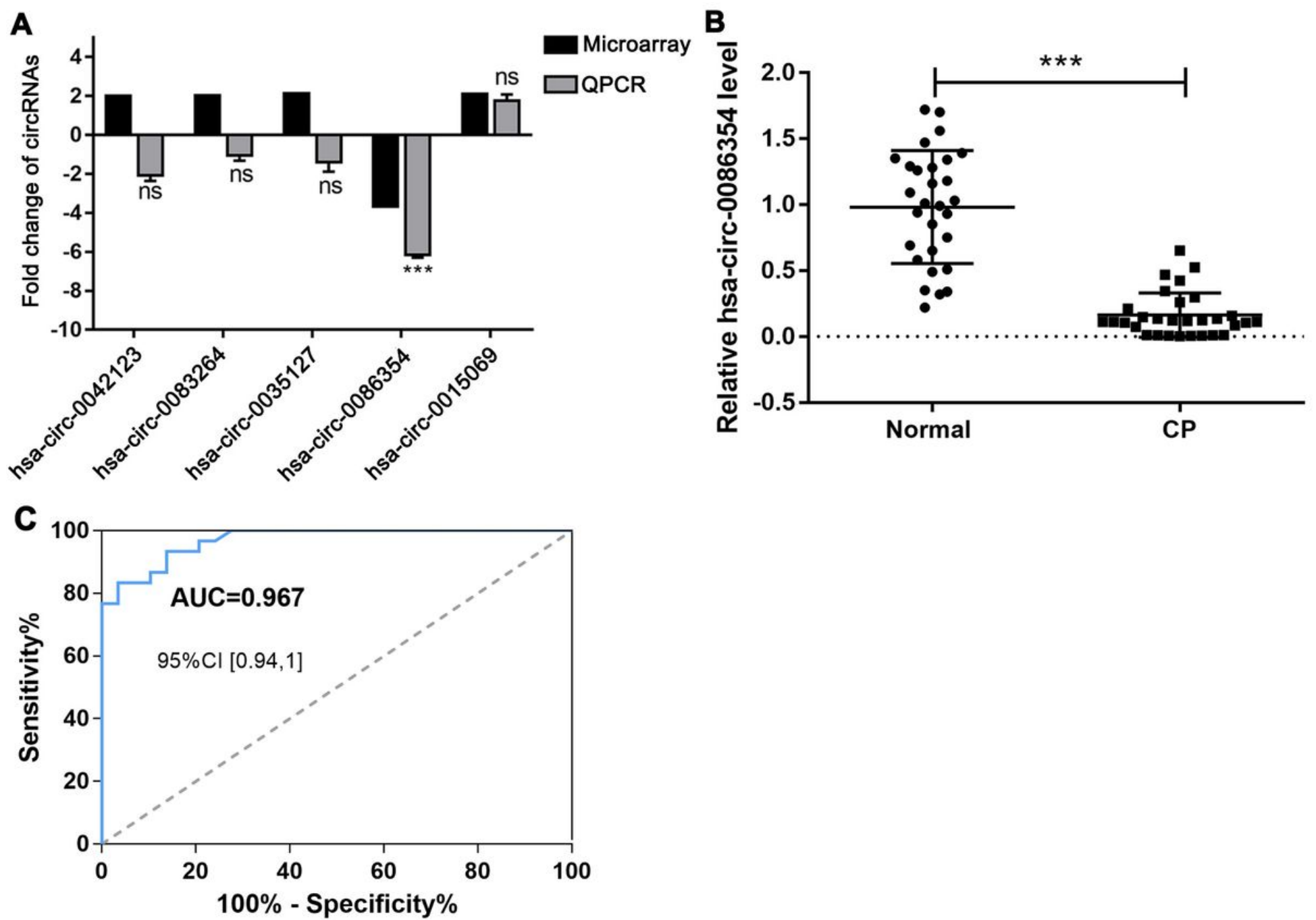

Figure 2

Hsa_circ_0086354 is a potential biomarker for early diagnosis of CP. Five circRNAs screened out by microarray was further validated by quantitative real-time PCR. (A) All expression levels in Control group were normalized to " 1 ". Fold changes of CP vs. Control obtained from microarray and real-time PCR were shown. (B) Relative expression of hsa_circ_0086354 in 30 pairs of CP and Control samples detected by quantitative real-time PCR was shown. (C) ROC curve analysis was carried out to assess the value of hsa_circ_0086354 as biomarker for CP diagnosis. The AUC was 0.967 , the sensitivity was 0.833 and the specificity was 0.966 . "ns": not significant; $\star \star \star p<0.001$. CP: cerebral palsy; ROC: receiver operating characteristic; AUC: area under the curve. 
A

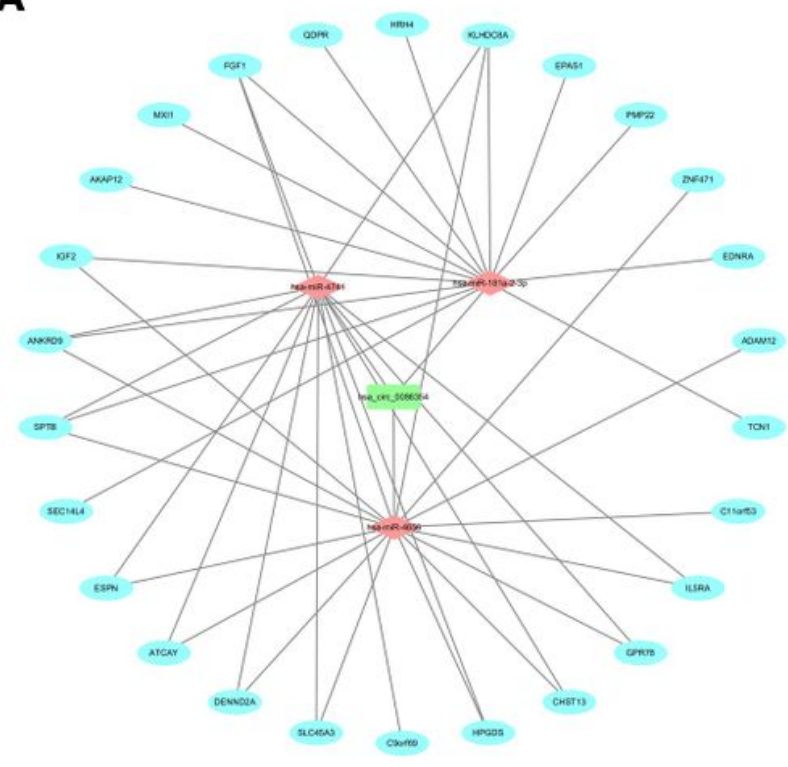

B

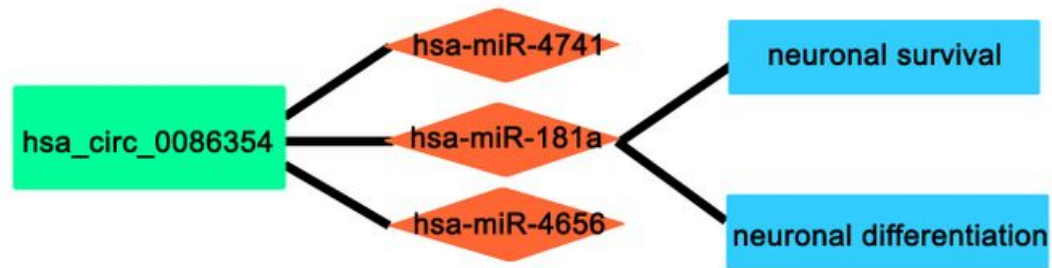

\section{Figure 3}

Hsa_circ_0086354 is involved in neuronal survival and neuronal differentiation via targeting miR-181a. (A) Cytoscape analysis was performed to show the hsa_circ_0086354 associated ceRNA network. (B) A schematic diagram about circRNAs, microRNAs and CP pathologic mechanisms. ceRNA: competitive endogenous RNA; CP: cerebral palsy. 